\title{
The surgical treatment of peptic ulcer
}

\author{
IAIN E. GiLleSPIE \\ Department of Surgery, Western Infirmary, Glasgow, W.1
}

\section{Indications for elective operation}

It is generally agreed that the commonest reason for advising a patient with a peptic ulcer to have an operation is the failure of conservative medical measures to control the symptoms adequately, and this applies both to duodenal and gastric ulcers. Few patients are considered for surgical treatment before the clinician is satisfied that the measures outlined by Dr Langman have been conscientiously applied, and that, in spite of these measures, relapses of typical ulcer dyspepsia become more frequent, more severe and more prolonged. It is difficult to lay down hard and fast rules, such as a definite length of history beyond which automatically operation should be advised. Patients vary considerably in the periodicity of their ulcer dyspepsia, and any individual patient may himself vary in the frequency and severity of relapses from year to year. From large reported series such as those of Thoroughman \& Ginglich (1963) and Kemp (1967), it is evident that the majority of duodenal ulcer patients coming to operation have had symptoms for between 5 and 10 years, and an appreciable proportion have a history going back more than 20 years. Factors other than simply the length of time over which symptoms have persisted are generally taken into consideration in reaching a decision for operation in a particular patient.

When a patient complains that antacids which formerly gave satisfactory relief are no longer effective, it seems reasonable to consider operative treatment without undue delay even if the history is relatively short. It seems unreasonable to insist on waiting a statutory period of time if the patient has tried a large variety of antacid medications in adequate dosage without obtaining pain relief. On the other hand, the patient who enjoys long remissions of up to a year at a time and can fairly readily control his relapses when they recur by a simple antacid preparation may well never be advised to have surgical treatment unless some complication develops.

Loss of time from work because of ulcer symptoms is an important factor to take into consideration, particularly in the case of young and middle-aged men with families dependent on their continuing in regular employment. Repeated interruption of sleep by the pain, radiation of the pain through to the back, and the onset of vomiting, particularly if it be of a retention nature are all factors which generally influence a decision towards surgery. A strong family history of peptic ulceration, particularly in the patient's immediate relatives may well lead to operation at an earlier stage than in those without such a strong family history. The previous occurrence of either a perforation or an episode of haemorrhage from the ulcer will also make it more likely that the patient will eventually require definitive surgery for the ulcer and are, therefore, additional weighting factors.

The question of malignant change only enters into consideration in the case of gastric ulcero and it is virtually unknown for a chronic duo denal ulcer to become malignant. We have two difficulties when it comes to the possible relationship between benign gastric ulcer and carcinoma of the stomach. First there is the question of how frequently an ulcer which starts as a benign lesion later changes into a malignant one. While the development of frank carcinoma in one segment of an otherwise benign ulcer is undoubted, the variation in the reported incidence of this occurrence is quite considerable. However, it is generally held to be infrequent. The second problem is that of being certain of the diagnosis on clinical, radiological and even gastroscopic examination. For these two reasons we are perhaps more ready to advise operation in patients with gastric ulcer than in the case of the more common duodenal ulcer, and certainly where any diagnostic doubt exists early operation would seem indicated.

Finally with regard to blood-groups, Dr Lang man, who has contributed so much information on this subject, and recently reviewed the entire position (Langman, 1967), has shown that the patients of group $O$, particularly those who are unable to secrete the blood-group secretor substances in the gastric mucus are more likely to require surgical treatment than those belonging to other groups. I think it is fair to state, however, 
that there is not sufficient evidence at present to make the blood-group status influence a decision regarding elective surgical treatment.

\section{Type of operation}

Since the surgical approach to the treatment of different types of ulcer differs in some important respects, these will be considered separately.

\section{Duodenal ulcer}

The common aim of most elective operations for duodenal ulcer is permanently to reduce the capacity of the stomach to secrete acid and pepsin, in the belief that the hypersecretion of these substances is causally related to the ulcer. While this assumption remains unproved there is no doubt that in the majority of instances the measures taken to reduce the acid and pepsin secretion do result in healing of the ulcer and protection against further ulceration.

A brief consideration of the mechanisms stimulating the acid and pepsin-producing cells shows that there are several possible surgical means of diminishing these secretions. First of all we can divide the vagus nerves and thereby prevent the transmission of nervous impulses to the stomach, provided that we are technically able to interrupt all the vagal fibres. Secondly, the source of the hormone gastrin, namely the pyloric antral mucosa, can be excised, to remove the main mechanism by which the physical presence of food in the stomach stimulates gastric secretion. Thirdly, it might be felt desirable to combine both of these procedures and perform vagotomy plus resection of the pyloric antrum. Fourthly, we are also able to reduce acid-pepsin secretion by removing a substantial proportion of the acid and pepsin-secreting cells themselves.

There is considerable evidence that in duodenal ulcer patients there is an increase in vagally controlled cholinergic activity, and for this reason vagotomy with a simple drainage procedure is currently in vogue as the elective operation of choice of many surgeons. The drainage procedure is required to accompany the vagotomy since vagal denervation alone has been found to lead to undue gastric stasis and vomiting. The two drainage procedures most widely employed are gastrojejunostomy and pyloroplasty. Although theoretically pyloroplasty might seem a more sound physiological procedure, it is not as yet apparent whether one holds distinct advantages over the other. There are also differences of opinion among surgeons regarding the superiority of one particular type of pyloroplasty. The Heineke-Mikulicz procedure involves simply the making of a longitudinal incision through the pyloroduodenal area and a transverse closure of this, and the Finney procedure comprises approximating the greater curvature of the distal antrum with the medial wall of the proximal duodenum and making a two-layer closure similar to a gastrojejunostomy. Again, provided that the calibre of the resultant opening is sufficiently large, there is no evidence that one form of pyloroplasty is distinctly superior to the other.

Removal of the pyloric antrum alone has generally been found inadequate to protect the patient against further ulcer recurrence, and this may be taken to indicate that disordered antral function, although it may occur, is not a frequent major aetiological factor in duodenal ulcer.

From the relatively few available reports, it seems, as might be expected, that a combination of vagotomy with antrectomy results in a much greater reduction in acid and pepsin secretion than either procedure alone, and this is apparently accompanied by an extremely low reported incidence of ulcer recurrence.

There are two factors contributing to the reduction of gastric acid and pepsin secretion following partial gastrectomy. The first is of course the complete removal of the pyloric antrum, the main source of gastrin, and since it has been argued that this alone is generally insufficient to prevent ulcer recurrence in duodenal ulcer patients, the second aim, namely to remove a considerable proportion of the acidpepsin cells, is believed to be the more important one. There are, as we know, two possible means of reconstruction after the gastrectomy is performed. Clinical experience has shown that the gastro-duodenal anastomosis of the Billroth I procedure is followed by a much higher incidence of recurrent ulcer than the gastrojejunal anastomosis of the Polya type of operation, and this latter, or one of its many modifications, is generally regarded as the procedure of choice when partial gastrectomy is practised. It is not clear why there is this marked difference in recurrent ulceration following two basically similar procedures, but there are several possible theoretical explanations, among them the simple one that a more radical removal of the gastric tissue is possible with the Polya type of gastrectomy. With regard to the actual technique of the Polya gastrectomy, there is considerable difference of opinion as to the value of a valve in the anastomosis, and this would seem largely to be a matter of the individual surgeon's preference.

How are we to decide which of the various procedures just outlined is the one of choice for a particular duodenal ulcer patient? There would 
seem to be three factors to be taken into consideration. The first is the expected operative mortality, the second the incidence of recurrent ulcer, and the third the incidence of the various early and long-term post-gastric-surgery sequelae. All surgeons must surely envy the admirable zero mortality record from the excellent study here in our host city, but we must also look at the available mortality statistics from the world's published literature to get as representative as possible a view of what is general experience. Table 1 compares the early mortality rates from vagotomy with gastrojejunostomy, vagotomy plus antrectomy, and both types of partial gastrectomy pooled from the available English language literature in 1961 by Welbourn \& Johnston. Vagotomy with the drainage procedure was found to have the lowest mortality rate, partial gastrectomy the highest, and the combined vagotomy plus antrectomy an intermediate level, suggesting that it was the actual gastric resection with its attendant anastomotic lines which posed the risk. Thus solely on the grounds of safety to the patient from threat to life there would seem to be an argument in favour of vagotomy with a simple drainage procedure. However, there is now a much greater experience of vagotomy plus drainage than was available in 1961 , and we may well find that the average mortality rate from this procedure is a little higher than was found in 1961. A recent review of 374 of our own patients who underwent vagotomy with gastrojejunostomy between eight and ten years ago (Battersby, 1967 personal communication) has shown an immediate postoperative mortality of $1.3 \%$

TABLE 1

The surgical treatment of duodenal ulcer; immediate mortality and recurrent ulcer rate (from Collected Data-Welbourn \& Johnston, 1961)

\begin{tabular}{|c|c|c|}
\hline Operation & $\begin{array}{l}\text { Immediate } \\
\text { mortality }\end{array}$ & $\begin{array}{l}\text { Recurrent } \\
\text { ulcer rate }\end{array}$ \\
\hline $\begin{array}{l}\text { Polya gastrectomy } \\
\text { Billroth I gastrectomy } \\
\text { Vagotomy + gastrojejunostomy } \\
\text { Vagotomy + antrectomy }\end{array}$ & $\left\{\begin{array}{l}1 \cdot 7 \\
0.66 \\
2 \cdot 2\end{array}\right\}$ & $\begin{array}{l}1 \cdot 4 \\
8 \cdot 5 \\
1 \cdot 8 \\
0 \cdot 4\end{array}$ \\
\hline
\end{tabular}

Turning to the question of recurrent ulcer, it is much more difficult to obtain reliable figures, in view of the difficulty in making the diagnosis on clinical, radiological and even on occasions operative examination. It is almost certain that although the recurrent ulcer figures shown in Table 1 for the two types of partial gastrectotomy, and for vagotomy plus antrectomy would still apply today, a much higher figure would now be given for vagotomy with gastrojejunostomy, or with the other drainage procedure, pyloroplasty. There would seem thus a much greater risk of further ulceration following this procedure. The proven recurrence rate in our own 8- to 10-year follow-up series is $6 \%$, and there are other patients in whom the diagnosis, though not substantiated, is likely. Most, if not all of these recurrences are due to the vagotomy being incomplete, and this is perhaps the most important obstacle to the uniform acceptance of vagotomy. Unfortunately, our means of assessing the degree of completeness of vagal section still leave something to be desired. However, such evidence as is available suggests that the incidence of incompleteness may sometimes be as high as $30 \%$. To summarize from the point of view of recurrent ulcer alone, it would seem that the combination of vagotomy with antrectomy would provide the greatest degree of protection.

The third consideration is that of the disorders of absorption, nutrition and blood, and the postprandial symptoms which may be a legacy of the surgical procedure. The severity and complexity of these various abnormalities vary consider? ably, and it is generally considered that partia禹 gastrectomy is responsible for a greater propor tion of such disorders than the other operations It may well be that further experience will confirm this differential, but it is well to remember that we have a much more extensive knowledge of the late sequelae of partial gastrectomy than we do of the more recently popular vagotomy procedures. Again from the analyses by Battersby of the 8- to 10-year follow-up of vagotomy with gastrojejunostomy, there was a $3.6 \%$ incidence of early surgical complications, and an overall incidence of $18 \%$ of patients classified as in the unsatisfactory group IV according to the Goligher modification of Visick's grading scheme. It still remains to be seen whether there will eventually be a marked difference in the incidence of post-operative sequelae after vagotomy with a simple drainage procedure, as compared with gastric resection.

Perhaps the most widely discussed complication to follow vagotomy is diarrhoea. There is great variation in the reported occurrence and severity of this symptom in different series. A major difficulty is the definition of the symptom. Most surgeons find that although there is a general tendency towards increasingly frequent bowel motions following vagotomy with drainage, this amounts to disability only in a very small proportion of patients. It has been claimed 
that the alteration in bowel habit is related to the vagal denervation of the abdominal organs other than the stomach, and various forms of selective vagotomy have been introduced in recent years to overcome this theoretical disadvantage. There is some accumulating evidence that several patterns of selective vagotomy do in fact adequately denervate the stomach, but further careful studies will be required to ascertain that the selective procedure gives adequate protection against recurrent ulceration, and does in fact diminish the incidence of the other sequelae, including the diarrhoea.

Not too much is known about the incidence of early and late post-operative sequelae from vagotomy plus antrectomy, and while on the one hand the limited resection might be expected to be followed by a lower incidence of troublesome complications than after the more radical gastrectomy, on the other hand the patients might be prone both to the complications of resection and those following vagal denervation.

To summarize, it would at present appear that partial gastrectomy is likely to carry a slightly greater mortality rate and higher incidence of early and late surgical complications, although the difference in incidence of both from those of vagotomy with a simple drainage procedure is not as great as was believed 5 or 10 years ago. Partial gastrectomy on the other hand would appear to give a better guarantee against recurrent ulceration than vagotomy plus drainage. Vagotomy plus antrectomy probably carries the least risk of recurrent ulceration of all the procedures, but the mortality risk and possibly the incidence of sequelae are probably intermediate between those of the other two procedures. Many surgeons appear to feel that if the mortality rate differential continues in favour of vagotomy with drainage, they would prefer this operation, and try to overcome the problem of the greater number of recurrent ulcers.

Use of gastric secretion results in selection. Although the idea of using the level of gastric acid response to help in the selection of one type of operation as opposed to another is an interesting one, being pursued by several groups of investigators, there is at present, no clear indication that the knowledge of a patient's secretory response should influence the recommendation for surgical treatment, or choice of procedure.

\section{Gastric ulcer}

The most widely practised operation for benign gastric ulcer is probably still a Billroth I partial gastrectomy including removal of the ulcer, but aiming to conserve more of the stomach than in a gastrectomy for duodenal ulcer. This policy is influenced by the almost invariable absence of hypersecretion, and the belief that since pyloric antrum dysfunction may be causally related to the ulcer, an important feature of the operation is complete removal of the antrum. The Billroth I partial gastrectomy for gastric ulcer is not followed by the high incidence of recurrent ulcer found when it is used in the treatment of duodenal ulcer. In recent years several workers have reported series of gastric ulcers treated by vagotomy with drainage, drainage procedures alone, simple wedge excision of the ulcer, and distal gastrectomy leaving a high-sited ulcer still in place. Favourable results have been reported from each of the procedures, although perhaps the least encouraging reports have been from the drainage procedures alone. It is generally agreed that it is more difficult to assess progress in patients with gastric ulcer, in view of the more widely varied natural course, when compared with duodenal ulcer. It is certainly of great importance that these various procedures be fully assessed in the treatment of gastric ulcer, as they may well throw considerable light on the pathology of the disease. It is, however, probably too early to determine whether any of these alternative operations is more satisfactory in the surgical management of gastric ulcer than the generally accepted Billroth I partial gastrectomy.

\section{Zollinger-Ellison syndrome}

The rarity of this condition should first be emphasized. The clinical suspicion is raised by either an unusually severe dyspeptic history, the radiological finding of multiple ulcers, the accompanying symptom of profuse diarrhoea, or, if gastric acid secretion measurements are made, the finding of an unusually large acid-output without exogenous stimulation, followed by a very small further increase on giving a secretory stimulant in maximal dosage. Theoretically excision of the pancreatic tumour which is responsible for the excessive quantitites of gastrin should be sufficient to cure the disorder. Unfortunately however, it is known that approximately $50 \%$ of these tumours will metastasize and display other signs of malignancy so that one cannot always give the guarantee that removal of the pancreatic tumour alone will not be followed by recurrence of the disease at a later date. It is, therefore, usually recommended that in addition to resecting the portion of pancreas bearing the tumour, if this is technically feasible, it is wise to perform total gastrectomy. This 
somewhat radical procedure would appear to be necessary, since even a small residual cuff of acid-secreting mucosa may be stimulated to such an extent by the tumour deposits as to cause life-threatening recurrent ulceration. Even in the presence of known metastases the total gastrectomy is still worth considering since the metastases often grow quite slowly and the patients may still survive for many years. Several surgeons have reported small series of patients treated by pancreatic resection alone and no gastric procedure, and follow-up observations over several years have shown these patients to remain in apparent good health. However, we are at present unable to determine which of the patients with the Zollinger-Ellison syndrome have potentially malignant lesions, and the risks both of perforation and severe bleeding from large and multiple recurrent ulcers are appreciable. For this reason a strong case can be made out for performing the total gastrectomy on establishing the diagnosis.

\section{Stomal ulcer}

The management of recurrent ulcer can pose difficult problems. The majority of these occur after elective operations for duodenal ulcer, and the following are suggested procedures should further surgical exploration be required. In the belief that an important disorder in duodenal ulcer is vagal overactivity, it would seem reasonable to perform vagotomy when the ulcer follows a partial gastrectomy. Should the previous gastrectomy have been a Billroth I type, it would also seem wise to convert this to Polya procedure.
It is believed that most recurrent ulcers following vagotomy with a drainage procedure are due to incompleteness of the vagotomy, and a re-exploration of the oesophagus either through the transthoracic route or preferably through the abdomen may well reveal an obvious missed trunk or strand of fibres. Theoretically the division of any missed nerve fibres should give adequate further protection, and if the surgeon is confident that he in turn has not missed any other strands, this procedure alone might suffice. With insulin evidence of complete vagotomy, or uncertainty about the further oesophageal exploration, it would seem wise to add a limited gastric resection ensuring complete removal of the antrum. In the Zollinger-Ellison syndrome, which might well be suspected from the rapidity and severity of recurrence following a previous operation, exploration of the pancreas, the removal of any tumour found therein, and total gastrectomy would seem indicated.

\section{References}

KEMP, D. (1967) An evaluation and comparison of the early and late results of standardized Polya gastrectomy. Gut, 8, 151.

LANGMAN, M.J.S. (1967) The medical treatment of peptic ulceration. Hosp. Med. 1, 383.

Thoroughman, J.C. \& Ginglich, G.W. (1963) Results of Surgery for Peptic Ulcer (Co-operative Study by Twelve Veterans Administration Hospitals) (Ed. by R. W. Postlethwait and J. C. Thoroughman), p. 109. Saunders, Philadelphia.

Welbourn, R.B. \& Johnston, I.D.A. (1961) The assessment and selection of elective operations for peptic ulceration. British Surgical Practice: Surgical Progress, pp. 301-331. Butterworth's, London. 